\title{
New Aspects of Structural and Magnetic Behaviour of Martensites in Ni-Mn-Ga Alloys
}

\author{
Volodymyr Chernenko ${ }^{1}$, Victor L'vov ${ }^{2}$, Eduard Cesari ${ }^{3}$, Jaume Pons ${ }^{3}$, Richard Portier ${ }^{4}$ \\ and Sergei Zagorodnyuk ${ }^{2}$ \\ ${ }^{1}$ Institute of Magnetism, Vernadsky str.36-b, Kiev 03142, Ukraine \\ ${ }^{2}$ Taras Shevchenko University, Kiev 03127, Ukraine \\ ${ }^{3}$ Departament de Física, Universitat de les Illes Balears, E-07071 Palma de Mallorca, Spain \\ ${ }^{4}$ CECM(CNRS), F-94497 Vitry-sur-Seine and ENSCP, F-75231 Paris Cedex 05, France
}

\begin{abstract}
The structural changes and magnetic anomalies accompanying martensitic transformations in Ni-Mn-Ga alloys are briefly discussed. The role of lattice tetragonality of martensite in the reduction of magnetic field needed for the observation of large magnetostrain effect is theoretically analyzed, considering the compensation of the magnetic anisotropy. The possibility of the field reduction is based on the previously observed lattice parameter dependence on the temperature and proper fit of the alloy specimen shape. The model shows that a significant reduction of the magnetic field needed for the giant MSE can take place in martensites with $0.98<c / a<1.04$.
\end{abstract}

(Received December 6, 2001; Accepted February 15, 2002)

Keywords: nickel-manganese-gallium alloys, martensitic transformation, tetragonality of martensitic lattice, magnetostrain effect, magnetic anisotropy, phenomenological model

\section{Introduction}

The Ni-Mn-Ga alloys showing a thermoelastic martensitic transformation (MT) form a relatively new family of shape memory and superelastic materials. ${ }^{1-3)}$ The alloys with compositions near their prototype $-\mathrm{Ni}_{2} \mathrm{MnGa}-$ are ferromagnetic Heusler L $2{ }_{1}$ ordered alloys with Curie temperature $T_{\mathrm{C}}$ around $370 \mathrm{~K}^{2)}$ The coupling of the structural and magnetic degrees of freedom gives new possibilities for the unusual magnetomechanical behaviour of martensite, like a large magnetostrain effect, which enhances the interest in these alloys to be applied as so called magnetic shape memory materials. $^{4-7)}$ It has been shown that the martensitic structure in $\mathrm{Ni}-\mathrm{Mn}-\mathrm{Ga}$ alloys can be controlled not only by the external uniaxial stress and temperature ${ }^{1)}$ but also by composition variation ${ }^{8)}$ being related to the electron/atom ratio. ${ }^{9)}$ According to the transformation behavior and relative values of $T_{\mathrm{M}}$ and $T_{\mathrm{C}}$ temperatures $\left(T_{\mathrm{M}}\right.$ is the martensitic transformation temperature), both being functions of electron concentration, ${ }^{9)} \mathrm{Ni}-\mathrm{Mn}-\mathrm{Ga}$ alloys can be subdivided into different groups. ${ }^{2,9)}$ Detailed structural studies have been performed for the alloys with $T_{\mathrm{M}}>T_{\mathrm{C}}$, using X-ray diffraction (XRD) and transmission electron microscopy (TEM), ${ }^{8)}$ thus the symmetry and lattice parameters of unit cells for the martensites encountered in these alloys have been clarified. ${ }^{3,8)}$ Neutron and X-ray powder diffraction measurements of near stoichiometric $\mathrm{Ni}_{2} \mathrm{MnGa}$ alloy with $T_{\mathrm{M}}<T_{\mathrm{C}}$ were undertaken in Refs. 10,11). TEM results are also reported for these alloys, (see e.g., Ref. 8)). All the aforementioned studies reveal the tetragonal unit cell of martensites formed as a result of distortion of the initial austenitic cubic lattice. In case of the alloys with $T_{\mathrm{M}}<T_{\mathrm{C}}$ this distortion is characterized by the lattice parameters ratio $c / a<1$ and is accompanied by shuffling of close-packed planes with periodicity of 5 planes (five-layered martensite). In the alloys with $T_{\mathrm{M}}>T_{\mathrm{C}}$ the basic martensitic structure is tetragonal with $c / a>1$; other possible structures can be obtained by microtwinning of the basic one. ${ }^{8)}$ Thus, it may be concluded that among all the structural changes accompanying the MT in Ni-Mn-Ga alloys the tetragonal distortion play the key role and $c / a$ ratio is one of the important parameters influencing magnetic and electronic properties of the alloys. ${ }^{12)}$ Magnetic moments, anisotropy constants and exchange interactions are sensitive to the lattice distortions and atomic environment. In the case of $\mathrm{Ni}-\mathrm{Mn}-\mathrm{Ga}$ alloys they have been shown to be essentially different for austenitic and martensitic states. ${ }^{1,13-15)}$ Moreover, it has been experimentally confirmed that the martensitic structures encountered in $\mathrm{Ni}-\mathrm{Mn}-\mathrm{Ga}$ alloys affect their magnetic properties. $^{16)}$

In this paper emphasis will be given to certain details of the relationship between the crystallographic peculiarities of martensite and its magnetic characteristics. In particular, a possible influence of $c / a$ value on the magnitude of magnetostrain effect for a given magnetic field amplitude will be highlighted.

\section{Experimental Procedure}

Several Ni-Mn-Ga alloys with different compositions were prepared by induction melting and casting. In this paper we will present some results obtained in two alloys with the following compositions: $\mathrm{Ni}_{51.2} \mathrm{Mn}_{31.1} \mathrm{Ga}_{17.7}$ (alloy 1) with $T_{\mathrm{M}}=446 \mathrm{~K}$ and $T_{\mathrm{C}}=356 \mathrm{~K}$, and $\mathrm{Ni}_{49.2} \mathrm{Mn}_{26.6} \mathrm{Ga}_{24.2}$ (alloy 2 , single crystalline form) with $T_{\mathrm{M}}=173 \mathrm{~K}$ and $T_{\mathrm{C}}=368 \mathrm{~K}$. X-ray diffraction (Siemens D5000), TEM (Hitachi H600, $100 \mathrm{kV}$ ) and HREM (Jeol 2011, $200 \mathrm{kV}$ ) were used for structural studies. The thin foils for TEM/HREM observations were prepared by twin jet polishing in a solution of $30 \%$ nitric acid in methanol at $240 \mathrm{~K}$. The martensitic state in alloy 1 was investigated at room temperature, while the cooling stage (only available for TEM) was used to study the martensite of alloy 2 . 


\section{Results and Discussion}

The XRD pattern obtained from the alloy 1 in bulk condition is shown in Fig. 1(a). This pattern fits with a non-modulated tetragonal cell with lattice parameters $a=$ $0.544 \mathrm{~nm}$ and $c=0.662 \mathrm{~nm}$. This basic unit cell is built in the same crystallographic axes as the parent phase (cubic L $2_{1}$ structure with $a_{\mathrm{c}}=0.583 \mathrm{~nm}$ ); therefore, during the martensitic transformation the cubic unit cell shrinks in two axes and expands in the third one. TEM observations performed in the same alloy reveal the presence of zones with the socalled 7-layered martensite, recognized by the six extra spots along a particular $\langle 110\rangle$ direction (Fig. 1(b)). Other long periodic martensitic structures (with periods of 8 and 10 planes) coexisting with non-modulated tetragonal lattice can also be observed in the alloys with $T_{\mathrm{M}}>T_{\mathrm{C}}$, depending on their previous thermomechanical treatments. ${ }^{3,8)}$ These structures are usually described as stackings of nearly close-packed planes derived from $\{110\}_{\text {aust }}$ planes, and they can also be interpreted as a microtwinning mode of the basic non-modulated tetragonal cell. ${ }^{8)}$ Such interpretation is confirmed by recent HREM observations, at least for the 7-layered martensite, as it is shown in Fig. 2. The picture shows the boundary between a region of non-modulated tetragonal phase (A) and a microtwinned one (B). The diffraction pattern corresponding to the microtwinned part is like that shown in Fig. 1(b), although the selected area is much bigger than that shown in the HREM image. It is worth to note that, in the microtwinned zone, the thickness of the twins is not perfectly periodic, and in some cases the stacking sequence of nearly close-packed planes is fairly far from the $(5 \overline{2})_{2}$ one, which is typically used to model such structure. ${ }^{8)}$ This is more clear in the enlarged part of the image. Thus, it is concluded that the stacking sequence is in average periodic with a period of 7 planes, but locally it can be severely disturbed. This also explains the diffuse intensity visible in the diffraction patterns along the direction of modulation (Fig. 1(b)).

The alloys with $T_{\mathrm{M}}<T_{\mathrm{C}}$ exhibit the so-called 5-layered martensite, showing diffraction patterns characterized by four equally-spaced extra spots along the $\langle 110\rangle$ direction. ${ }^{1,8)}$ The basic unit cell (not considering the modulation) is tetragonal with $c / a<1$. However, in the present alloy 2 (with low transformation temperature), the selected area diffraction patterns (Fig. 3) are slightly different. Indeed, four extra spots can be distinguished in between two consecutive fundamental ones, but the extra spots are not equally spaced. In addition, other extra weak spots are visible in between those more intense four extra spots. The structural models developed to interpret the 5-layered modulated martensite ${ }^{1,8)}$ cannot explain the features of the pattern shown in Fig. 3. More work is currently done in order to interpret such incommensurate diffraction patterns.

The results related to the tetragonality ( $c / a$ ratio) of martensitic phase in $\mathrm{Ni}-\mathrm{Mn}-\mathrm{Ga}$ being available from our present and former works $8,10,11,17,18)$ are plotted in Fig. 4 as a function of electron concentration $e / a$. Although there is a lack of systematic measurements of $c / a$ ratio for the alloys with the values $e / a \leq 7.7(e / a=7.7$ corresponds to the crossing of $T_{\mathrm{C}}(e / a)$ and $T_{\mathrm{M}}(e / a)$ lines on the phase diagram $^{9)}$ ) one might assume after inspection of Fig. 4 that in this

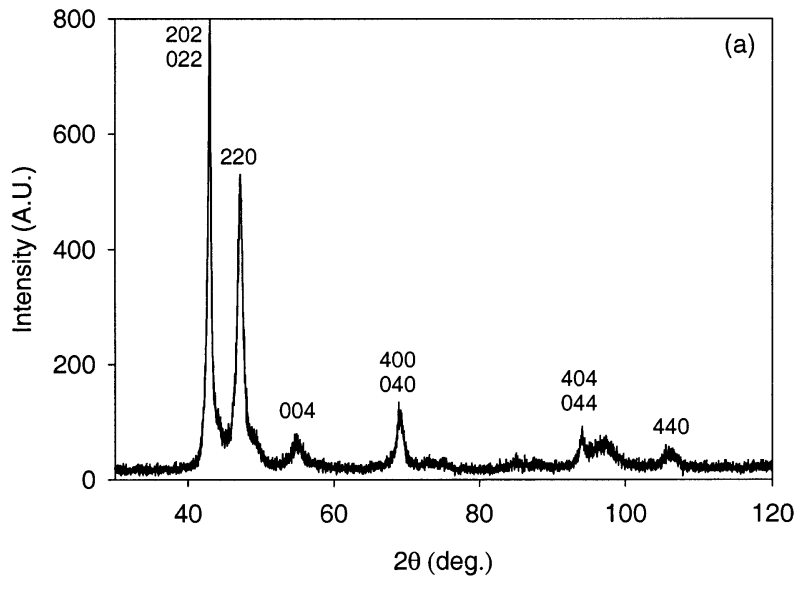

(b)

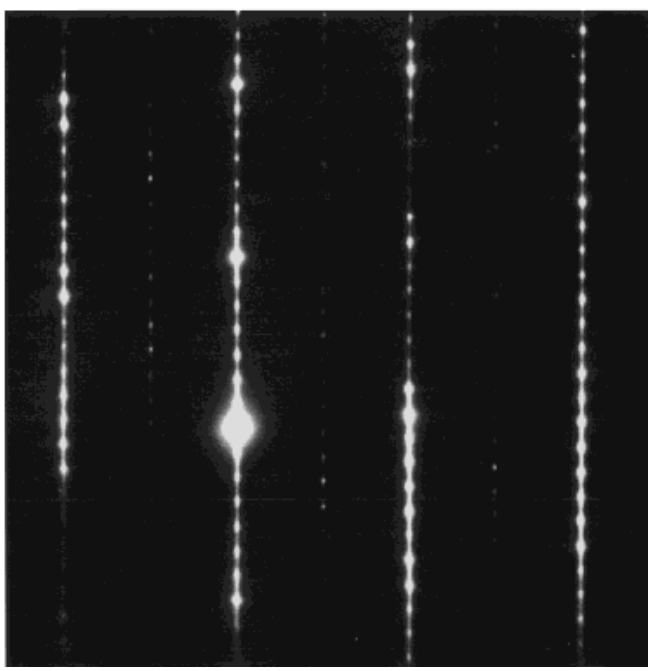

Fig. 1 XRD (a) and electron diffraction (b) patterns obtained from alloy 1 at room temperature.

critical range $c / a$ experiences an abrupt change from $c / a<1$ to $c / a>1$. Alongside with this change, the monotonous and roughly linear concentration dependence of $c / a$ is observed in the region where $c / a>1$. In other words, the transformation anisotropic strain is an increasing function of $e / a$ ratio. In addition, the tetragonality is temperature dependent, as it was experimentally shown in Ref. 11). The lattice parameters of martensite in $\mathrm{Ni}_{2} \mathrm{MnGa}$ compound monotonously become closer to the lattice parameter of cubic parent phase upon approaching $T_{\mathrm{M}}$, so that, for instance, $c / a=0.946$ at $4.2 \mathrm{~K}$, while $c / a=0.969$ at $T=200 \mathrm{~K} \approx T_{\mathrm{M}} \cdot{ }^{11)}$ Concerning Fig. 4, it has to be pointed out that the measurements in $\mathrm{Ni}-$ $\mathrm{Mn}-\mathrm{Ga}$ alloys with $c / a>1$ were done at room temperature (which is lower than $T_{\mathrm{M}}$ by $80 \mathrm{~K}$ or more), while those with $c / a<1$ were done either at room temperature, i.e. within transformation hysteresis loop for an alloy with $e / a \approx 7.56^{8)}$ or at temperatures more than $100 \mathrm{~K}$ below $T_{\mathrm{M}}$ for alloys with $e / a \approx 7.50$. $^{7,11,17)}$

The $c / a$ ratio can affect the magnetic properties and magnetoelastic behavior of martensite significantly. First of all, the tetragonal martensites with $c / a<1$ correspond to the easy-axis type of ferromagnetic ordering while the alloys with $c / a>1$ most probably will be the ferromagnets of the easyplane type. ${ }^{13)}$ The fact that $c / a$ in Ni-Mn-Ga can be regulated to be close to 1 offers new opportunity for the observation of 


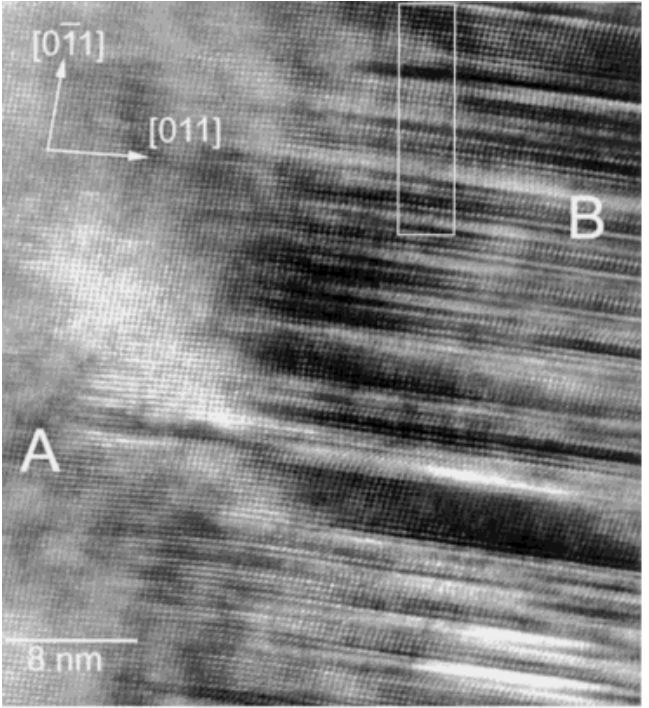

(a)

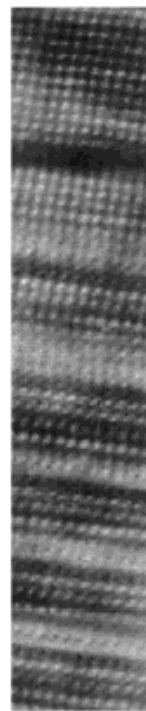

(b)

Fig. 2 (a) Low magnification HREM image of martensite in alloy 1. (b) enlargement of the framed zone. A: region of non-modulated tetragonal phase. B: region showing lattice microtwinning.

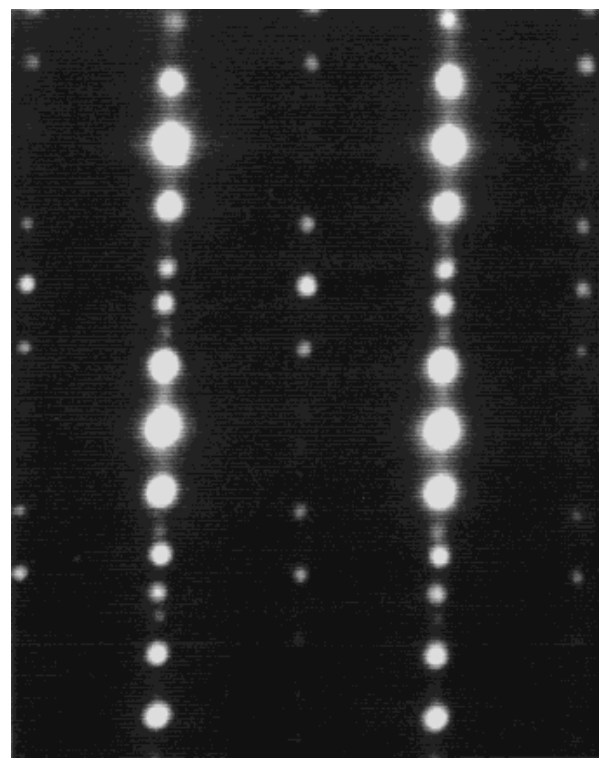

Fig. 3 Electron diffraction pattern obtained from alloy 2 at liquid nitrogen temperature.

fairly large magnetostrain effect (MSE) in a comparatively small applied magnetic field, as will be shown below. The increase of magnetoelastic response observed at temperatures inside the transformation region ${ }^{7,19)}$ confirms this opportunity. It is worth to note that in most of the works devoted to the MSE measurements, the martensitic structure is not specified. To our knowledge, the numerical value of $c / a$ ratio of the martensite giving giant MSE is stated only in several works (see, e.g. Refs. 4-7)), being $c / a \approx 0.94$. A lack of information about MSE exists for the alloys with $c / a>1$.

The works dealing with the giant magnetostrain effect in the $\mathrm{Ni}-\mathrm{Mn}-\mathrm{Ga}$ alloy system are mainly focused on the enlargement of the field-induced deformations. Nevertheless, the possibility of reduction of the magnetic fields needed for observing large enough MSE is also important for applications. This possibility arises from the obvious interrelation

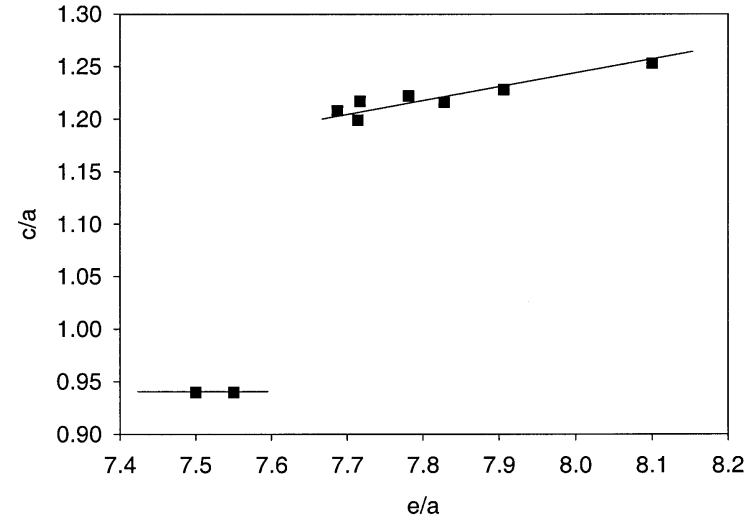

Fig. 4 Plot of tetragonality ratio $(c / a)$ of martensite in different $\mathrm{Ni}-\mathrm{Mn}-\mathrm{Ga}$ alloys as a function of the electron concentration.

between the magnetization curves $M(H)$ and the field dependencies of strain $\varepsilon(H)$ observed by many authors. In particular, it is seen from the existing experimental data that both $M(H)$ and $\varepsilon(H)$ curves reach saturation at the same magnetic field value $H=H_{\mathrm{S}}$. Thus, the problem of reduction of the magnetic saturation field $H_{\mathrm{S}}$ arises. A theoretical value of this field can be found from the expression for the non-isotropic part $F$ of the magnetic energy. For the tetragonal martensitic phase

$$
F=A m_{x}^{2}+M^{2}(\boldsymbol{m} \cdot \boldsymbol{D} \cdot \boldsymbol{m}) / 2-\boldsymbol{m} \cdot \boldsymbol{H} M
$$

where the first term describes the magnetic anisotropy energy of crystal lattice with the $c$-axis oriented in $x$-direction, the second and third terms present the magnetostatic and Zeeman energies respectively, $\boldsymbol{D}$ is the demagnetization matrix, and $\boldsymbol{m}=\boldsymbol{M} / M$. A phenomenological model of ferromagnetic martensite ${ }^{13)}$ relates the magnetic anisotropy constant $A$ to the magnetoelastic constant $\delta_{1}$ characterizing a cubic parent phase, and the lattice parameters $a, c$ of tetragonal phase. It is convenient to present this relationship in the form $A=6 \delta_{1}(1-c / a)$ (see Refs. 13,14)). As far as $\delta_{1}<0$ in zero magnetic field the $\boldsymbol{m}$ vector of a Ni-Mn-Ga martensite with $c / a$ value about 0.94 reported in Refs. 10,18 ) is parallel to $c$-axis. ${ }^{13,15)}$

Let the specimen be an ellipsoid of revolution with the revolution axis aligned with $y$-direction. In such a case $\boldsymbol{D}$ is a diagonal matrix with the elements $D_{1}=D_{3} \neq D_{2}$. A standard minimization procedure for the energy given in eq. (1) shows that the magnetic field applied in $y$-direction causes the vector $\boldsymbol{m}$ rotation in $x y$ plane by an angle $\psi=\arccos \left(H / H_{\mathrm{S}}\right)$, and hence, a magnetic saturation takes place when

$$
H=H_{\mathrm{S}}=M\left[D_{2}-D_{1}+12|\delta|(1-c / a)\right]
$$

where $\delta=\delta_{1} / M^{2}$.

Equation (2) being valid when $H_{\mathrm{S}} \geq 0$ shows that the mutual compensation of the magnetic anisotropy of the crystal lattice and the anisotropy of the specimen caused by the magnetostatic energy is possible. A condition of complete compensation is $H_{\mathrm{S}}=0$, i.e.

$$
c / a=1-\left(D_{1}-D_{2}\right) / 12|\delta|
$$

Thus, the compensation of magnetic anisotropy can be observed in two cases: i) when $c / a<1, D_{1}-D_{2}>0$ 


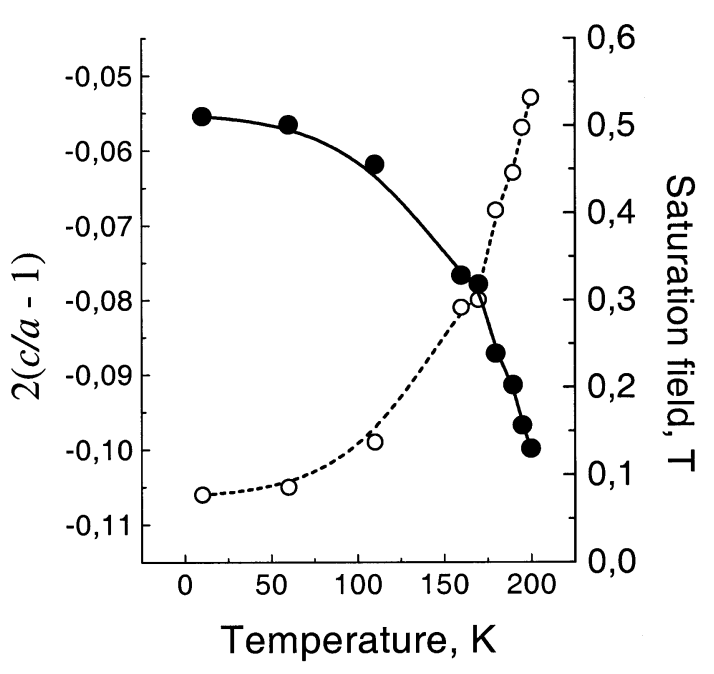

Fig. 5 The order parameter value (open circles) and the magnetic saturation field (full circles) vs. temperature for the elongated ellipsoid-shaped specimen of $\mathrm{Ni}_{2} \mathrm{MnGa}$ alloy with the length/width ratio equal to 4 .

(oblate tetragonal cell, oblong specimen); ii) when $c / a>1$, $D_{1}-D_{2}<0$ (oblong tetragonal cell, oblate specimen). The limiting values of $D_{1}-D_{2}$ are $-4 \pi$ (for the thin platelet) and $+2 \pi$ (for the long rod). The estimation $\delta_{1}=-1.2 \mathrm{MPa}$ obtained in Refs. 13), 14) for the alloy with $M \approx 700 \mathrm{G}$ results in the value $\delta \approx-23$. Substitution of these values into the eq. (3) leads to the conclusion that the complete compensation of the magnetic anisotropy is possible in the martensitic alloys with $0.98<c / a<1.04$, i.e. $-0.08<u_{0}<0.04$, where $u_{0}=2(1-c / a)$ is an equilibrium value of the order parameter of cubic-tetragonal phase transformation. For these alloys a complete field-induced reorientation of the martensite variant with $c$-axis directed along $x$ into the variant with $c$-axis parallel to $y$ results in the field-induced deformations $-0.04<\varepsilon_{\mathrm{xx}}<0.02$ and $-0.02<\varepsilon_{\mathrm{yy}}<0.04$. It should be expected therefore, that a substantial reduction of the magnetic field needed for the observation of the field-induced strains $\varepsilon>1 \%$ is possible for the "compensated" specimens.

For the $\mathrm{Ni}_{2} \mathrm{MnGa}$ compound the $u_{0}(T)$ and $H_{\mathrm{S}}(T)$ functions can be obtained using eq. (2) and the $c(T)$ and $a(T)$ dependencies measured in Ref. 11). The results are presented in Fig. 5. The $H_{\mathrm{S}}(T)$ plot shows, that the complete compensation of two contributions to the magnetic anisotropy is not achievable for the alloy studied in Ref. 11), but the fivefold decrease of the saturation field of martensite is possible in the vicinity of austenite start temperature.

The almost complete field-induced reorientation of martensite is essentially irreversible. As far as the reversibility of MSE is a point of crucial importance, the martensitic alloys showing reversible field-induced strains $\varepsilon_{\mathrm{ii}}<0.01$ are studied intensively. A phenomenological model of magnetoelastic behavior of such alloys ${ }^{13,14,20)}$ enables the theoretical description of magnetization curves and MSE. In particular, the averaged magnetization and macroscopic longitudinal strain, induced by the field aligned with y direction are expressed as: ${ }^{13,14,21)}$

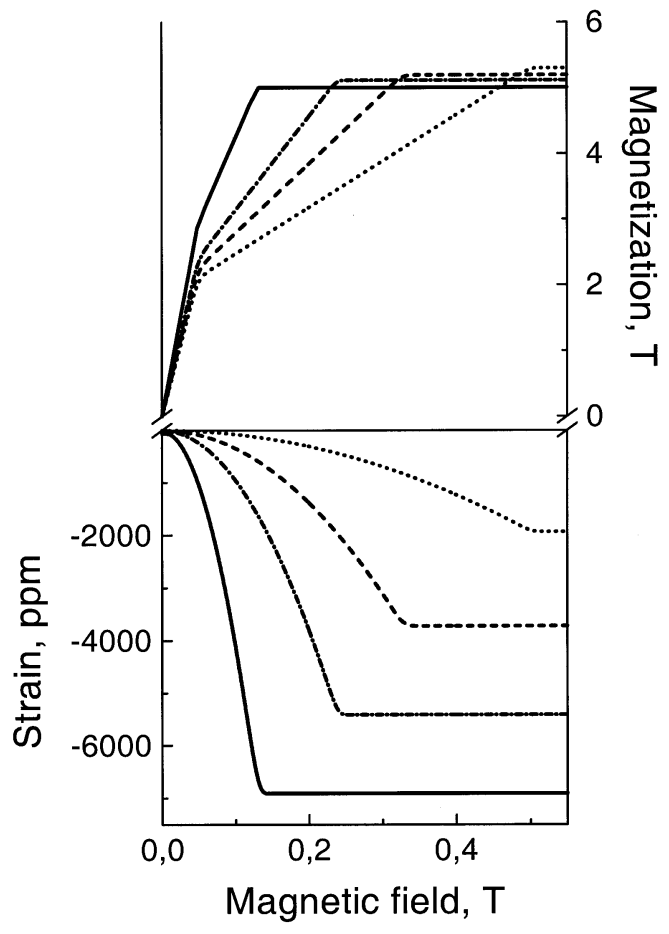

Fig. 6 Computed field dependences of averaged magnetization and field induced strains in Ni-Mn-Ga martensite as a function of magnetic field for $T=60 \mathrm{~K}, \partial \sigma / \partial \varepsilon=4 \mathrm{GPa}$ (doted line) $T=160 \mathrm{~K}, \partial \sigma / \partial \varepsilon=2 \mathrm{GPa}$ (dashed line); $T=180 \mathrm{~K} \partial \sigma / \partial \varepsilon=1.3 \mathrm{GPa}$ (dot-and-dashed line); $T=200 \mathrm{~K} \partial \sigma / \partial \varepsilon=1 \mathrm{GPa}$ (solid line).

$$
\begin{aligned}
\langle M(H, T)\rangle & =M(T)\left[\frac{1}{3} \Delta(H, T)+\frac{2}{3} \cos \psi(H, T)\right] \\
\varepsilon(H, T) & =6\left(\partial \varepsilon / \partial \sigma_{\mathrm{yy}}\right) \delta M^{2}(T) \cos ^{2} \psi
\end{aligned}
$$

where the derivative $\partial \varepsilon / \partial \sigma_{\mathrm{yy}}$ characterises the anomalous elastic compliance of the martensite in $y$ direction, $\Delta(H, T)=H / D_{2} M(H, T)$ for $H<H_{\mathrm{S}}, \Delta(H, T)=1$ for $H \geq H_{\mathrm{S}}$, and the $M(T)$ function satisfies the well-known equation $M(T)=M(0) \tanh \left[T_{\mathrm{C}} M(T) / T M(0)\right]$. The magnetization curves and magnetic-field-induced strains computed from eq. (4) are presented in Fig. 6. The substantial decrease of the anomalous elastic stiffness $\partial \sigma_{\text {yy }} / \partial \varepsilon$ value for the temperatures approaching $T_{\mathrm{M}}$ has been tentatively considered in the course of computations. Figure 6 clearly shows the above-mentioned correlation between magnetization and field-induced strain in martensite. The results of computations agree qualitatively with the experimental results reported in Refs. 7), 19). Figure 6 illustrates also the possibility of saturation of $\varepsilon(H)$ for comparatively low magnetic fields (about $0.1 \mathrm{~T}$ ).

Another possibility for the field reduction arises from the observation of giant MSE within the martensitic transformation temperature interval $\Delta T=T_{\mathrm{Ms}}-T_{\mathrm{Mf}}$ of mixed twophase state, ${ }^{7)}$ where $T_{\mathrm{Ms}}$ and $T_{\mathrm{Mf}}$ are the temperatures of the start and finish of martensitic transformation, respectively. In this case a strain of $\varepsilon>1 \%$ can be induced by a comparatively weak magnetic field in the specimens with small $\Delta T \leq 10 \mathrm{~K}^{20)}$ 


\section{Conclusions}

(1) Different martensitic structures based on tetragonal lattice have been obtained in $\mathrm{Ni}-\mathrm{Mn}-\mathrm{Ga}$ alloy system. In particular HREM observations of alloys with $c / a>1$ confirm fine microtwinned nature of martensitic structure with 7-layer periodicity and averaged (5⿳亠丷厂) stacking sequence, although with areas severely distorted.

(2) The tetragonality ratio of martensitic lattice in ferromagnetic Ni-Mn-Ga alloys affects the sign and value of their magnetic anisotropy, so that the amount of giant MSE can be controlled by this ratio as well.

(3) A possibility of significant reduction of the magnetic field necessary for giant MSE has been theoretically shown to exist in martensites having $0.98<c / a<1.04$.

\section{Acknowledgements}

V.A.C. is grateful to the Universitat de les Illes Balears (UIB, Spain) and to Université Pierre et Marie Curie (Paris, France) for financing his stays at these Universities. The TEM and HREM observations have been performed at the Serveis Cientificotècnics of the UIB. Partial financial support from DGI (projects PB98-0127 and MAT2000-0862-C03-02) is gratefully acknowledged.

\section{REFERENCES}

1) V. A. Chernenko and V. V. Kokorin: Proceedings of the Int. Conf. on Martensitic Transformations (ICOMAT-92), (Monterey Inst. for Adv. Studies, Monterey, California, USA, 1993) pp. 1205-1210.

2) V. A. Chernenko, E. Cesari, V. V. Kokorin and I. N. Vitenko: Scr. Metall
Mater. 33 (1995) 1239-1244.

3) V. A. Chernenko, C. Seguí, E. Cesari, J. Pons and V. V. Kokorin: Phys Rev. B57 (1998-I) 2659-2662.

4) K. Ullakko, J. K. Huang, C. Kantner, V. V. Kokorin and R. C. O'Handley: Appl. Phys. Lett. 69 (1996) 1966-1969.

5) R. D. James and M. Wuttig: Philos. Mag. A 7 (1998) 1273-1299.

6) S. J. Murray, M. A. Marioni, A. M. Kukla, J. Robinson, R. C. O'Handley and S. M. Allen: J.Appl. Phys., 87 (2000) 5774-5776.

7) V. A. Chernenko, V. A. L'vov, M. Pasquale, S. Besseghini, C. P. Sasso and D. A. Polenur: Int. J. of Applied Electromagnetics and Mechanics 12, Ns. 1, 2 (2000) 3-8.

8) J. Pons, V. A. Chernenko, R. Santamarta and E. Cesari: Acta Mater. 48 (2000) 3027-3038.

9) V. A. Chernenko: Scr. Mater 40 (1999) 523-527.

10) P. J. Webster, K. R. A. Ziebeck, S. L. Town and M. S. Peak: Philos. Mag. B49 (1984) 295-306.

11) Y. Ma, S. Awaji, K. Watanabe, M. Matsumoto and N. Kobayashi: Solid State Commun. 113 (2000) 671-676.

12) V. V. Godlevsky and K. M. Rabe: Phys. Rev. B63 (2001) 134407.

13) V. A. L'vov, E. V. Gomonaj and V. A. Chernenko: J. Phys.: Condens. Mat. 10 (1998) 4587-4596.

14) V. A. Chernenko, V. A. L'vov and E. Cesari: J. Magn. Mag. Mat. 196197 (1999) 859-860.

15) R. Tickle and R. D. James: J. Magn. Mag. Mat. 195 (1999) 627-638.

16) V. A. Chernenko, A. Amengual, E. Cesari, V. V. Kokorin and I. K. Zasimchuk: J. de Phys. IV, Col. C2, 5 (1995) 95-98.

17) A. Zheludev, S. M. Shapiro, P. Wochner, A. Schwartz, M. Wall and L. E. Tanner: Phys. Rev. B51 (1995) 11310-11314.

18) I. K. Zasimchuk, V. V. Kokorin, V. V. Martynov, A. V. Tkachenko and V. A. Chernenko: Phys. Metall. Metall. 69 (1990) 104-108.

19) C. H. Yu, W. H. Wang, J. L. Chen, G. H. Wu, F. M. Yang, N. Tang, S. R. Qi, W. S. Zhan, Z. Wang, Y. F. Zheng and L. C. Zhao: J. Appl. Phys. 87 (2000) 6292-6294.

20) V. A. Chernenko, V. A. L'vov, E. Cesari and P. McCormick: Mater. Trans., JIM 41 (2000) 928-932.

21) V. A. L'vov, S. P. Zagorodnyuk and V. A. Chernenko: submitted to Mater. Trans., (2001). 